\title{
Hiperplasia endotelial papilar intravascular oral: uma entidade rara
}

\section{Intravascular papillary endothelial hyperplasia: a rare entity}

João Augusto Vianna Goulart Filho'; Karuza Maria Alves Pereira²; Hébel Cavalcanti Galvão

\section{unjtermos resumo}

Hiperplasia endotelial papilar

vascular

Angiomatose intravascular

Hemangioendotelioma

intravascular vegetante de

Masson
A hiperplasia endotelial papilar intravascular (HEPI) é uma lesão vascular reativa caracterizada pela proliferação endotelial excessiva localizada no interior de vasos sangüíneos comumente dilatados, associada a trombos em organização, ou secundária a outras lesões vasculares, como hemangiomas e granulomas piogênicos. A HEPI é um achado incomum na cavidade oral, onde os lábios são o principal sítio de acometimento, e surge clinicamente sob a forma de nódulos azulados de aspecto clínico semelhante ao de lesões como hemangioma, mucocele e varicosidades. Sob o aspecto histopatológico, observam-se projeções papilares de tecido conjuntivo fibroso revestidas por uma ou duas camadas de células endoteliais no interior de um lúmen vascular. A principal peculiaridade da HEPI reside em sua semelhança histológica com o angiossarcoma e na possível interpretação errônea como neoplasia maligna. Neste artigo, os autores descrevem um caso de HEPI oral e realizam uma breve revisão da literatura, enfatizando suas características histopatológicas e o diagnóstico diferencial. abstract

Intravascular papillary endothelial hyperplasia (IPEH) is a reactive vascular lesion characterized by excessive endothelial proliferation within a vascular lumen of commonly dilated vessels, associated to organizing thrombi or even secondary to other vascular lesions, such as hemagiomas and pyogenic granulomas. Oral lesions of IPEH are uncommon, being lips the most frequent site, followed by, in decreasing frequency, tongue and buccal mucosa. Clinically, IPEH appears as bluish nodules that resembles clinical features of lesions as hemangioma, mucocele and varice. On histopathological examination, IPEH presents as papillary projections composed by a fibrous core lined by one or two layers of plump endothelial cells, without evidence of celullar pleomorphism, mitotic activity or necrosis. The mean peculiarity of IPEH is its histologic similarity to angiosarcoma and possible misinterpretation with malignant neoplasm. In this article, the author describe a rare case of oral IPEH and review of literature, emphasizing its histopathologic features and diferencial diagnosis. key words

Intravascular papillary

endothelial hyperplasia

Intravascular angiomatosis

Masson's vegetant

intravascular

hemangioendothelioma

\section{Introdução}

A hiperplasia endotelial papilar intravascular (HEPI) é uma lesão vascular reativa caracterizada por proliferação excessiva de células endoteliais ${ }^{(8,10,14,18,19)}$.

De uma forma geral, essa lesão é observada no interior do lúmen de vasos sangüíneos ${ }^{(1,19)}$, apresentando-se basicamente sob três formas: uma pura ou primária, localizada no interior de espaços vasculares dilatados (56\% dos casos); uma mista ou secundária, associada a lesões vasculares preexistentes, como hemangiomas, hamartomas vasculares, malformações arteriovenosas e granulomas piogênicos ( $40 \%$ dos casos); e a forma

1. Aluno de mestrado do Programa de Pós-Graduação em Patologia Oral da Universidade Federal do Rio Grande do Norte (UFRN)

2. Aluna de mestrado do Programa de Pós-Graduação em Patologia Oral da UFRN.

3. Professora-doutora do Programa de Pós-Graduação em Patologia Oral da UFRN.

Trabalho desenvolvido no Programa de Pós-Graduação em Patologia Oral da UFRN 
extravascular, que surge ocasionalmente no interior de um hematoma ( $4 \%$ dos casos) $)^{(2,5,9,13-15)}$.

Esta entidade foi primeiramente relatada por Masson ${ }^{(11)}$ como um processo neoplásico que consistia na obliteração do lúmen vascular devido a hiperplasia endotelial papilar associada a alterações degenerativas e necrose secundárias, que, em virtude de sua semelhança com o angiossarcoma, foi denominada hemangioendotelioma intravascular vegetante.

Desde então termos como angiomatose intravascular, pseudo-angiossarcoma de Masson, hemangioma de Masson, hemangioendotelioma intravascular vegetante de Masson foram utilizados para designar a condição( ${ }^{(3,6)}$.

$\mathrm{Na}$ atualidade, o termo HEPI é apropriadamente utilizado para descrever o caráter reativo da lesão $(2,6,8,9,14,18,19)$.

A presença de trombos em organização e a diminuição da velocidade do fluxo sangüíneo vascular parecem estar relacionadas à patogênese desta lesão, embora o verdadeiro estímulo para o seu aparecimento ainda seja desconhecido ${ }^{(9,14)}$.

A HEPI é encontrada numa faixa etária que varia de 9 a 80 anos, acometendo freqüentemente a derme e o tecido subcutâneo de regiões expostas a traumatismos, como os dedos e a região de cabeça e tronco, surgindo como massas superficiais firmes à palpação e de coloração azulada, resultante da dilatação e obstrução do espaço vascular, podendo eventualmente produzir aspectos clínicos que simulem uma neoplasia ${ }^{(1,8,10,13,14)}$.

Justificando o pequeno número de relatos publicados nessa localização ${ }^{(9,16)}$, tal lesão é excepcionalmente rara na cavidade oral, onde afeta indivíduos com média de 58 anos de idade ${ }^{(5)}$ e exibe maior predileção pela mucosa do lábio inferior, seguida, em ordem decrescente, por língua, lábio superior e mucosa jugal $(2,16,20)$. Alguns estudos referem uma predileção pelo sexo feminino ${ }^{(19)}$.

Outros sítios de acometimento incluem vasos da cavidade nasal, faringe, laringe, seios paranasais, canal auditivo interno, labirinto, válvulas cardíacas, mamas, trato gastrointestinal, fígado, rins, colo uterino, útero, uretra e pélvis ${ }^{(3,9,13)}$.

Devido ao fato de não envolver um aspecto clínico específico, é muito difícil estabelecer um diagnóstico prévio de HEPI antes da remoção cirúrgica da lesão ${ }^{(5,6,14)}$.

A semelhança histológica entre a HEPI e o angiossarcoma pode dificultar o diagnóstico diferencial entre essas duas lesões de tratamento e prognóstico completamente distintos, ressaltando a relevância clínica do exame histopatológico $^{(3,6,9,10,12,14,16,18)}$.
O presente artigo tem como objetivo relatar um caso de HEPI em região de cavidade oral, enfatizando suas características anatomopatológicas e a importância do diagnóstico diferencial para condições como o angiossarcoma.

\section{Relato do caso}

O paciente S.M.A., do sexo masculino, 28 anos de idade, apresentou-se ao Serviço de Diagnóstico Oral da Faculdade de Odontologia da Universidade Federal do Rio Grande do Norte (UFRN) tendo como queixa clínica a presença de uma massa amolecida indolor localizada no lábio superior.

Ao exame clínico, detectou-se a presença de uma lesão de aspecto nodular, coloração azulada e consistência mole à palpação, medindo cerca de $0,5 \mathrm{~cm}$ de diâmetro. Durante a anamnese, o paciente relatou história de trauma associado ao local da lesão. Duas semanas depois, o paciente foi submetido à biópsia excisional e a peça cirúrgica foi fixada em solução de formol a $10 \%$ e remetida ao Serviço de Patologia Oral da UFRN para a realização do exame histopatológico sob o diagnóstico clínico de hemangioma.

Os cortes histológicos corados através da técnica de hematoxilina e eosina (HE) revelaram a presença de proliferação endotelial de arranjo papilar confinada ao interior do lúmen vascular (Figura 1). As células endoteliais exibiam morfologia ovóide e formavam uma a duas camadas de células que revestiam delicados feixes de tecido conjuntivo fibroso, formando projeções de aspecto papilar (Figura 2).

As projeções papilares apresentavam-se interconectadas, formando ora um padrão mais sólido, ora um padrão disperso no interior do lúmen vascular, dando origem a uma ampla rede de espaços vasculares anastomosados semelhantes a capilares, ocasionalmente preenchidos por eritrócitos (Figura 3).

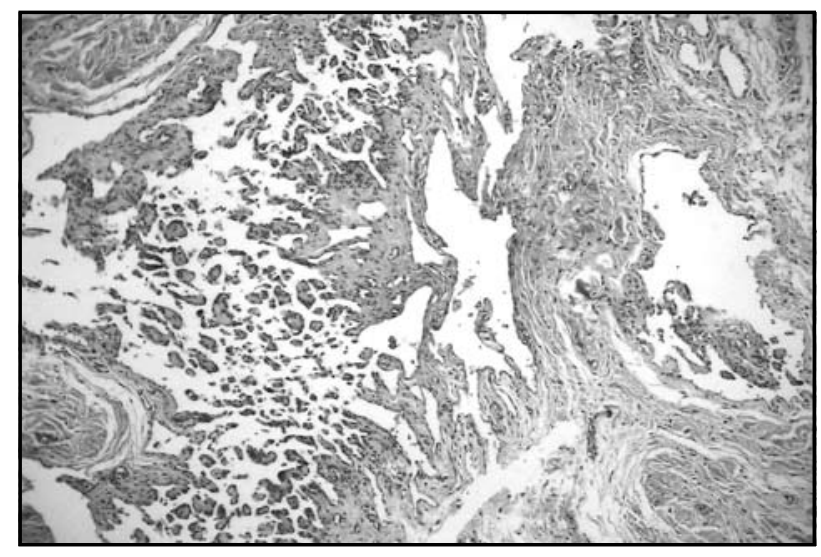

Figura 1 - Evidencia-se, em dois vasos sangüíneos, a proliferação intraluminal de células endoteliais arranjadas de forma papilar (HE 100x) 


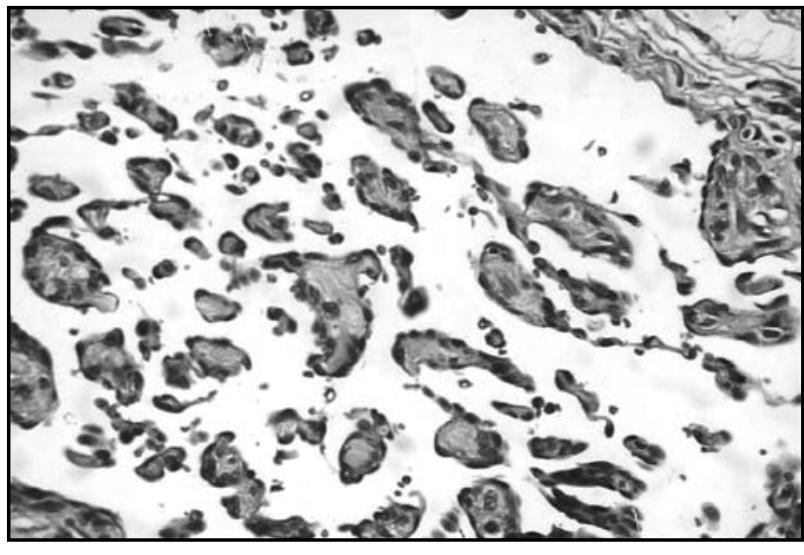

Figura 2 - Em maior aumento, nota-se a presença de papilas de tecido conjuntivo fibroso revestidas por uma camada de células endoteliais volumosas de aspecto normal (HE 400x)

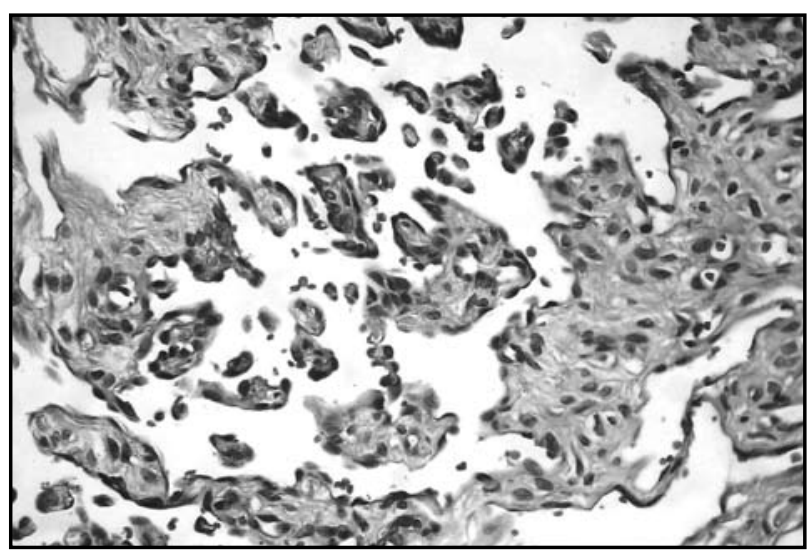

Figura 3 - Detalhe dos espaços vasculares semelhantes a capilares formando uma rede anastomosada, arranjados num padrão predominantemente sólido (HE 400x)

Em meio ao endotélio em proliferação, observaram-se pequenos e escassos fragmentos de material eosinofílico, cuja estrutura discretamente lamelar lembrava aquela constituinte do trombo em organização (Figura 4).

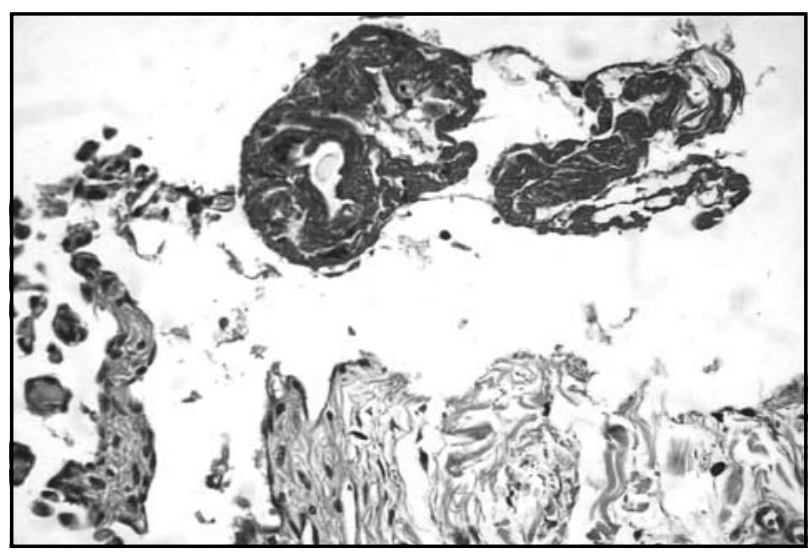

Figura 4 - Fragmento de material eosinofilico de organização lamelar semelhante ao observado em materiais tromboembólicos (HE 400x)
Não foi constatado qualquer indício de atipia celular, hipercromatismo, pleomorfismo celular, figuras de mitose, tampouco áreas de necrose ou invasão dos tecidos adjacentes, descartando-se a presença de um angiossarcoma.

$\mathrm{O}$ tecido conjuntivo fibroso adjacente era do tipo fibroso denso, contendo vasos de calibre e tamanho variados dentro dos aspectos de normalidade, e permeado por um discreto infiltrado inflamatório mononuclear, onde não se verificou a presença de qualquer outra lesão vascular. $\mathrm{O}$ revestimento epitelial era do tipo pavimentoso estratificado ortoceratinizado. A presença de lóbulos de glândulas sebáceas, fascículos nervosos e fibras musculares estriadas completava o quadro histológico.

Com base nos critérios e achados histológicos descritos, foi estabelecido o diagnóstico de hiperplasia endotelial papilar intravascular.

\section{Discussão}

Devido à freqüente associação com material tromboembólico(7), a HEPI passou a ser considerada uma proliferação endotelial reativa durante o processo normal de organização do trombo a partir de 1932, embora tal denominação só tenha sido difundida em 1976, por Clearkin e Enzinger ${ }^{(4,20)}$.

Alguns autores aventam que o trombo constitui uma matriz para a proliferação endotelial ${ }^{(11,17)}$, enquanto outros consideram que a associação do trombo em desenvolvimento com a presença da HEPI pode estar ligada a distúrbios hemodinâmicos locais ${ }^{(13)}$.

Não obstante, existem relatos da ocorrência de HEPI primária sem qualquer associação com a formação de trombo, outros em que a formação de trombo é secundária ao aparecimento da HEPI ou, ainda, a persistência da hiperplasia endotelial após o desaparecimento do trombo ${ }^{(10)}$.

A proliferação endotelial a partir de uma reação inflamatória crônica e/ou a irritação decorrente de traumatismo local também têm sido aceitas como possíveis agentes causais, embora permaneça incerta a verdadeira histogênese dessa patologi $\mathrm{a}^{(8,10)}$. No presente relato, o referido paciente apresentava história de trauma associado ao local da lesão, sugerindo a associação desse evento com o surgimento da lesão, embora o material trombótico evidenciado pareça constituir um achado secundário.

A proliferação endotelial excessiva estimulada pela produção local de fatores de crescimento tem constituído uma hipótese promissora para explicar a etiologia da $\mathrm{HEPI}^{(20)}$. Estudos através de northern blot e immunobloting 
revelaram aumento significativo da expressão do fator de crescimento fibroblástico beta (FGF- $\beta$ ), sugerindo a sua secreção elevada pelas células endoteliais através de um mecanismo autócrino ${ }^{(3)}$.

O caráter clínico inespecífico da HEPI impossibilita o seu reconhecimento imediato, já que outras lesões de aspecto azulado mais comuns da cavidade oral, como mucoceles, hemangiomas ou mesmo varicosidades, apresentam características clínicas idênticas ao exame clínico e geralmente constituem a hipótese diagnóstica inicial|(20), como observado no caso ora relatado. Sendo assim, o diagnóstico depende, sobretudo, do exame histopatológico, posto que exames de punção aspirativa por agulha fina (PAAF) são pouco sensíveis para a obtenção de um diagnóstico preciso ${ }^{(14)}$.

Em uma análise de 73 casos de HEPI oral, Tosios, Koutlas e Papanicolaou ${ }^{(19)}$ constataram uma evidente predileção pelo sexo feminino e indicaram lábios e língua como sítios de maior acometimento. Tal observação foi parcialmente evidenciada no presente caso, pois, apesar da presença da HEPI no lábio superior, o paciente era do sexo masculino.

Sob o aspecto histopatológico, a HEPI apresenta-se caracteristicamente como uma lesão bem circunscrita e constituída por estroma conjuntivo papilar de tecido conjuntivo fibroso, por vezes hialinizado, delimitado por duas ou mais camadas de células endoteliais volumosas, às vezes hipercromáticas, formando estruturas papilares que podem se fusionar e formar uma rede anastomosada de espaços vasculares ${ }^{(5,8,10,12,14,16,20)}$, tal qual observado no caso ora referenciado.

Eventualmente, a lesão pode apresentar um padrão celular sólido com focos de células endoteliais dispersos no tecido conjuntivo adjacente a zonas de ruptura da parede do vaso de origem, podendo, em alguns casos, simular a invasão tecidual observada no angiossarcoma $(1,2,5,6,10$, $12,14,16,19,20)$. No presente caso, observou-se um padrão sólido restrito ao interior do lúmen vascular, não havendo evidências de dispersão de células endoteliais no interior da lâmina própria.

A HEPI é diferenciada do angiossarcoma por não apresentar atipia celular, como pleomorfismo celular, atividade mitótica significativa, tampouco áreas de necrose ou padrão de crescimento invasivo, já que é descrita classicamente como uma lesão bem circunscrita ${ }^{(1-3,8,12,14,18-20)}$. No presente caso, a ausência de tais achados histopatológicos confirmou a natureza benigna da proliferação endotelial, levando-nos ao diagnóstico histopatológico de HEPI.

A excisão cirúrgica completa é o tratamento de escolha para a HEPI. A biópsia excisional geralmente é diagnóstica e curativa, refletindo um bom prognóstico para o paciente ${ }^{(14,}$ ${ }^{20)}$, como nesse relato de caso. São raros os casos de recorrências, comumente associados à remoção incompleta da lesão, especialmente as HEPI secundárias $(2,3,10,12,14,15,18,20)$.

\section{Referências}

I. AKHTAR, M. et al. Intravascular papillary endothelial hyperplasia of renal vein. Arch Pathol Lab Med, v. 129, p. 5 I6-9, 2005.

2. BUCHNER, A. et al. Oral intravascular papillary endothelial hyperplasia.J Oral Pathol Med, v. 19, n. 9, p. 419-22, 1990.

3. ÇAGLI, S. et al. Intravascular papillary endothelial hyperplasia of the central nervous system-four case reports. Neurol Med Chir, v. 44, n. 6, p. 302-10, 2004.

4. CLEARKIN, K. P.; ENZINGER, F.M. Intravascular papillary endothelial hyperplasia. Arch Pathol Lab Med, v. 100, p. 44I-4, 1976.

5. de COURTEN, A. et al. Intravascular papillary endothelial hyperplasia. Oral Dis, v. 5, n. 2, p. 175-8, 1999.

6. DUARTE, I. G. et al. Papillary endothelial hyperplasia presenting as chest wall neoplasm. Ann Thorac Sug, v. 67, p. 238-40, 1999.

7. HENSCHEN, F. L'endovasculite proliferante thrombopietique dans la lesion vasculaire locale. Ann Anat Pathol, v. 9, p. I I321,1932

8. HONG, S-G. et al. Intravascular papillary endothelial hyperplasia (Masson's hemangioma) of the liver: a new hepatic lesion.J Korean Med Sci, v. 19, p. 305-8, 2004.
9. IDE, F. et al. Rare vascular proliferations of the oral mucosa. Oral Med Oral Pathol Oral Radiol Endod, v. 97, p. 75-8, 2004.

I0. LEE, W.; HUI, F.; SITHO, Y.Y. Intravascular papillary endothelial hyperplasia in an intracranial thrombosed aneurysm: $3 T$ magnetic resonance imaging and angiographical features. Singapore Med J, v. 45, n. 7, p. 330-3, 2004.

I I. MASSON, P. Hemagioendotheliome vegetant intravascularie. Bull Soc Anat (Paris), v. 93, p. 517-25, 1923.

12. MATSUZAKA, K. et al. Intravascular papillary endothelial hyperplasia arising from the upper lip. Bull Tokyo Dent Coll, v. 44, n. 2, p. 55-9, 2003

13. MOON, W. S.; CHUNG, G. H.; HONG, K. H. Intravascular papillary endothelial hyperplasia in a vascular lesion of the maxillary sinus. Arch Pathol Lab Med, v. 124, p. I224-7, 2000.

14. NISHIMOTO, K. et al. Extravascular Endothelial hyperplasia arising from parapharyngeal space. Auris Nasus Larynx, v. 3I, p. 305-8, 2004.

15. PINS, M. R. et al. Florid extravascular papillary endothelial hyperplasia (Masson's pseudoangiosarcoma) presenting 
as a soft tissue sarcoma. Arch Pathol Lab Med, v. I17, p. 259-63, 1993.

16. RENSHAW, A. A.; ROSAI, J. Benign atypical vascular lesions of the lip: a study of 12 cases. Am J Surg Pathol, v. 17, n. 6, p. 557-65, 1993.

17. SALYER, W. R.; SALYER, D. C. Intravascular angiomatosis: development and distinction from angiosarcoma. Cancer, v. 36, p. 995-1001, 1975.

I8. STERN, Y. et al. Papillary endothelial hyperplasia in the tongue: a benign lesion that may be mistaken for angiosarcoma. J Otolaryngol, v. 23, n. 2, p. 8I-3, 1994.

1 9.TOSIOS, K.; KOUTLAS, I. G.; PAPANICOLAOU, S. I. Intravascular papillary endothelial hyperplasia of the oral soft tissues: report of 18 cases and review of the literature. J Oral Maxillofac Surg, v. 52, n. 12, p. 1 263-8, 1994.

20.TOKMAN, B. et al. Intravascular papillary endothelial hyperplasia of the mandibular lingual mucosa. Chin Med J, v. I 17, p. 1756-57, 2004. 